\title{
Tertiary lymphoid structures in the choroid plexus in neuropsychiatric lupus
}

\author{
Ariel D. Stock, ${ }^{1}$ Evan Der, ${ }^{1}$ Sivan Gelb, ${ }^{2}$ Michelle Huang, ${ }^{1}$ Karen Weidenheim, ${ }^{3}$ Ayal Ben-Zvi, ${ }^{2}$ \\ and Chaim Putterman ${ }^{1,4}$ \\ 'Department of Microbiology and Immunology, Albert Einstein College of Medicine, New York, New York, USA. \\ ${ }^{2}$ Department of Developmental Biology and Cancer Research, Faculty of Medicine, The Hebrew University, Ein-Kerem, \\ Jerusalem, Israel. ${ }^{3}$ Division of Neuropathology and ${ }^{4}$ Division of Rheumatology, Albert Einstein College of Medicine, \\ New York, New York, USA.
}

\begin{abstract}
The central nervous system manifestations of systemic lupus erythematosus (SLE) remain poorly understood. Given the well-defined role of autoantibodies in other lupus manifestations, extensive work has gone into the identification of neuropathic autoantibodies. However, attempts to translate these findings to patients with SLE have yielded mixed results. We used the MRL/ MpJ-Fas ${ }^{\text {Ipr/pr }}$ mouse, a well-established, spontaneous model of SLE, to establish the immune effectors responsible for brain disease. Transcriptomic analysis of the MRL/MpJ-Fas ${ }^{\mid p r / p r}$ choroid plexus revealed an expression signature driving tertiary lymphoid structure formation, including chemokines related to stromal reorganization and lymphocyte compartmentalization. Additionally, transcriptional profiles indicated various stages of lymphocyte activation and germinal center formation. The extensive choroid plexus infiltrate present in MRL/MpJ-Fas ${ }^{\text {pr//pr }}$ mice with overt neurobehavioral deficits included locally proliferating $B$ and $T$ cells, intercellular interactions between lymphocytes and antigen-presenting cells, as well as evidence for in situ somatic hypermutation and class switch recombination. Furthermore, the choroid plexus was a site for trafficking lymphocytes into the brain. Finally, histological evaluation in human lupus patients with neuropsychiatric manifestations revealed increased leukocyte migration through the choroid plexus. These studies identify a potential new pathway underlying neuropsychiatric lupus and support tertiary lymphoid structure formation in the choroid plexus as a novel mechanism of brainimmune interfacing.
\end{abstract}

Authorship note: $\mathrm{ABZ}$ and $\mathrm{CP}$ are co-senior authors.

Conflict of interest: The authors have declared that no conflict of interest exists.

Copyright: (c) 2019, American Society for Clinical Investigation.

Submitted: August 14, 2018

Accepted: April 23, 2019

Published: June 6, 2019.

Reference information: /CI Insight. 2019;4(11):e124203. https://doi. org/10.1172/jci.insight.124203.

\section{Introduction}

Systemic lupus erythematosus (SLE) is a complex, multifactorial autoimmune disease with diverse end-organ targets. The pathogenic mechanisms underlying many manifestations of SLE have been extensively characterized, with growing consensus regarding renal, vascular, and cutaneous presentations (1). Generally, these pathologies are attributable to autoreactive antibodies specific for tissue antigenic determinants and immune complex formation, coupled with downstream cell- and cytokine-mediated inflammation. The central nervous system (CNS) manifestations of SLE, though, have proved more challenging to understand. Signs and symptoms of neuropsychiatric SLE (NPSLE) are quite variable between patients, including focal manifestations closely related to lupus-associated hypercoagulability, as well as diffuse syndromes that include depression, anxiety, memory deficits, and general cognitive decline. The etiology of the diffuse cognitive and emotional manifestations of NPSLE is, however, poorly understood.

Several studies have found that extraneurally derived, neuropathic autoantibodies may be effectors of NPSLE, including anti-ribosomal-P (2) and anti-NMDA receptor antibodies (3). However, we recently showed that late reversal of systemic autoimmunity, including decreased autoantibody titers, does not fully prevent NPSLE in the classic MRL/MpJ-Fas ${ }^{\mathrm{pr} / \mathrm{pr}}$ (MRL/lpr) mouse model of SLE (4-6). Additionally, it is remarkable that certain features of NPSLE may precede diagnosis of SLE in both mouse models and human disease (7-9) and that systemic immunosuppression and symptomatic therapeutic modalities are inconsistently effective, suggesting that NPSLE may develop distinctly from SLE involvement in other organs as well as other neuropsychiatric diseases. 
Prevailing notions about the interface between the CNS and the immune system have undergone significant change in recent years. A novel mechanism of interstitial fluid clearance from the neuropil has been defined as the "glymphatic" system (10), which conducts cerebrospinal fluid flow from the subarachnoid space through the perivascular space of medium-sized vessels, bounded by the astrocytic foot processes of the neurovascular unit. Additionally, a recently rediscovered lymphatic system appears to reside within the dural sinuses (11), allowing for direct immunosurveillance and transmission of brain antigens to the deep cervical lymph nodes. Finally, the choroid plexus (CP), a cuboidal epithelial bilayer surrounding a highly vascularized capillary plexus, has recently been identified as an important interface for CNS immunosurveillance, within both homeostatic and inflammatory contexts. Specifically, it has been shown that the $\mathrm{CP}$ hosts a $\mathrm{CD} 4^{+} \mathrm{T}$ cell population demonstrating $\mathrm{T}$ cell receptor enrichment for neural antigens (12) and that during CNS infection (13) and experimental autoimmune encephalomyelitis (14), the CP serves as an important gateway for inflammatory responses.

The CP has historically been implicated in NPSLE as well, with evidence of IgG deposition and MRI findings suggestive of localized inflammation, though a causal relationship has not been demonstrated (1518). Similarly, in the MRL/lpr murine model of SLE, there is an extensive and progressive cellular infiltration within the CP that correlates with development of behavioral abnormalities (19). This infiltration has been previously characterized as mixed, with accumulations of predominately B and T lymphocytes, as well as plasma cells $(20,21)$. However, little has been done to carefully phenotype these brain-infiltrating lymphocytes in mice with lupus or in human disease or evaluate their role in disease pathogenesis.

Chronic inflammation, as it occurs in SLE, is conducive to the formation of tertiary lymphoid structures (TLSs) $(22,23)$. The diverse cytokine and chemokine repertoire found in chronically inflamed tissues, including upregulated lymphotoxin- $\alpha /-\beta(\operatorname{LT}-\alpha /-\beta)(24)$ and $\operatorname{CXCL13}(25,26)$, creates the conditions needed to generate these structures. Stromal LT receptor signaling is important in generating the microarchitecture required for efficient antigen presentation and follicle organization, which typically includes collagen-rich reticular cords that serve as conduits for cellular traffic. Similarly, CXCL13 is important in defining local B cell domains (27). Additionally, other cytokines and chemokines may contribute to the process of lymphoid follicle generation. Finally, certain cellular subsets are critical for the formation of ectopic lymphoid tissues with functional germinal centers (GCs), including B cells with GC activity, CD4 ${ }^{+} \mathrm{T}$ cell help provided by $\mathrm{T}$ follicular helper (Tfh) or Tfh-like cells, as well as antigen-presenting cells, be they tissue resident or peripherally derived (28).

Given the early development of neuropsychiatric relative to systemic manifestations of lupus, the relative resistance of NPSLE to systemic immunosuppression, and the existence of specific neuropathic antibodies, with our potentially novel findings described below, we conclude that the prominent mixed cellular infiltrate in the CP of mice with lupus represents a TLS, likely generated in response to signals that are both local CP epithelial derived as well as CNS derived. These TLSs potentially produce clonotypic responses to CNS antigens as a mechanism of bypassing the immune privilege of the CNS. Of broader significance, we report for the first time to our knowledge the presence of organized lymphoid structures within the $\mathrm{CP}$, providing another potential mechanism through which the immune system can interact with the CNS. Finally, transcriptome analysis revealed a unique expression signature in the CP of mice with lupus, consisting of the chemoattractant, organizational, and cell-phenotypical features of an organized TLS. These observations, coupled with evidence of leukocyte migration through the $\mathrm{CP}$ of patients with lupus, may provide for novel avenues of therapeutic intervention.

\section{Results}

The mixed cellular infiltrate in the $C P$ is structurally organized. We performed immunofluorescence evaluation of the $\mathrm{CP}$ infiltrate in 16- to 17-week-old female MRL/lpr mice as an initial screen of the leukocyte types present within the mixed infiltrate, as well as their organizational properties. In all cases, MRL/MpJ-Fas ${ }^{+/+}(\mathrm{MRL} /+)$ control mice were included, though these were uniformly negative for all leukocyte subtypes with the exception of an occasional $\mathrm{CD}^{+}$cell (Supplemental Figure 1A; supplemental material available online with this article; https://doi.org/10.1172/jci.insight.124203DS1). It is noteworthy that, with little exception, leukocyte infiltration into the $\mathrm{CP}$ of $\mathrm{MRL} / \mathrm{lpr}$ mice occurs with complete penetrance, both in our extensive experience as well as in that of others $(20,29,30)$. As a first indication of an organizational structure, we observed defined

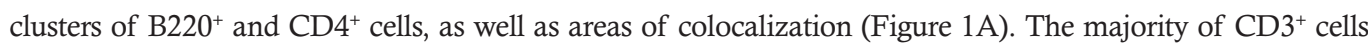
were $\mathrm{CD}^{+}$(Figure 1B), with less representation of $\mathrm{CD}^{+}$(Figure 1C). It is noteworthy that only rarely could 
A
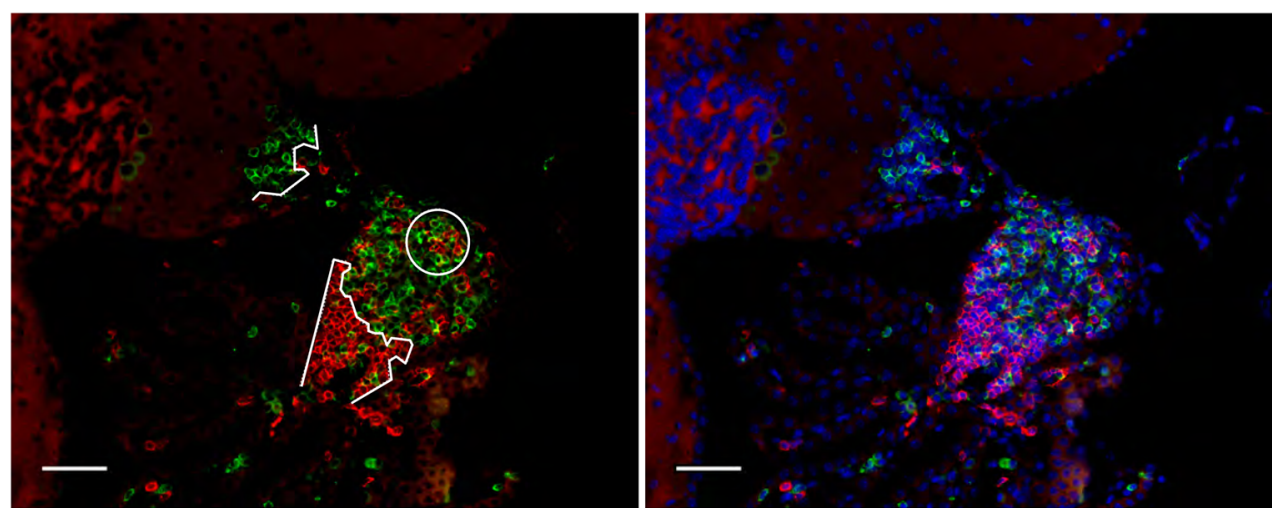

B

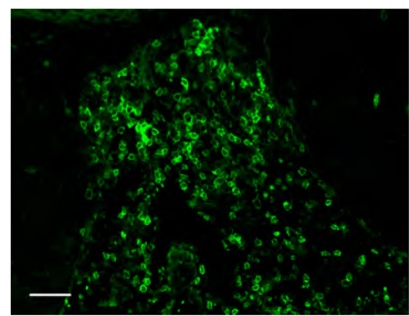

C

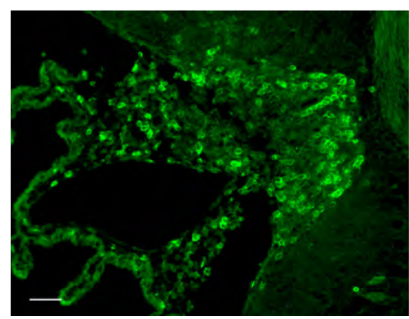

CD4

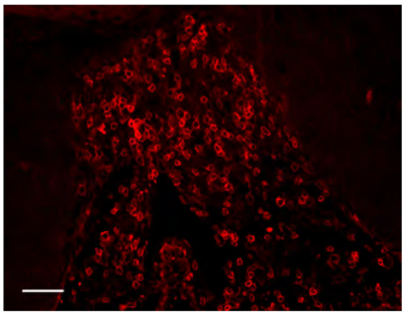

CD8

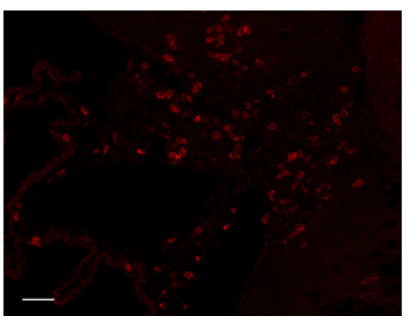

B220(red)/CD4(green)

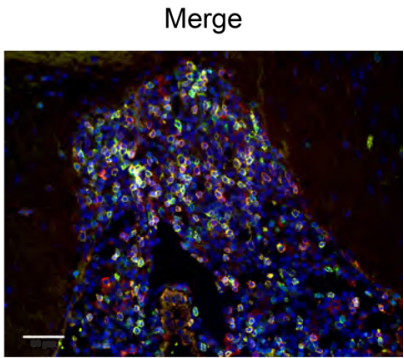

Merge

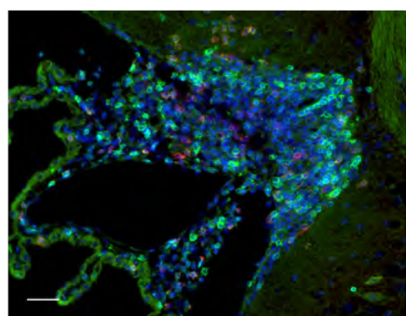

Figure 1. The cellular infiltrate in the CP is organized with a helper T cell dominance. CP tissue was isolated and stained as described in Methods. All subsequent figures show representative images from 16- to 17-week-old female $\mathrm{MRL} /$ Ipr mice (2 cohorts of $n=6$ mice). (A) B and T lymphocytes are readily identifiable as distinct (curved white lines) as well as interacting (white circle) cell clusters (left). DAPI counterstain is shown (right). (B) Most CD3+ CP-infiltrating T cells costain with $\mathrm{CD}^{+}$, with (C) only a minority expressing CD8 ${ }^{+}$. Scale bars: $50 \mu \mathrm{m}$.

we identify double-negative $\mathrm{T}$ cells, despite their dominance in the lymphoproliferative phenotype characteristic of the MRL/lpr strain (31-33). To identify extracellular features of the cellular infiltrate for clues as to what determines its organizational framework, we stained for reticulin, finding extensive organization of leukocytes around cord-like structures, which was confirmed by transmission electron microscopy (TEM) (Figure 2).

Elevated expression of lymphoid tissue inducing and regulating molecules in the $M R L / 1 p r C P$. We performed transcriptomic analysis on CP samples isolated from 12- to 16-week-old MRL/lpr and MRL/+ mice in an effort to correlate our histological findings with a transcriptional signature consistent with TLS formation. Age-matched murine lymphoid tissue (lymph node) from an unmanipulated non-autoimmune strain was analyzed as a positive control for lymphoid tissue-specific genes. When unsupervised hierarchical clustering was performed selecting the 200 most variable genes, several clusters of genes overlapped between MRL/+ and MRL/lpr CP but were absent from lymph nodes, indicating a set of CP-specific genes (Figure 3A). Interestingly, 2 sets of genes were expressed in both MRL/lpr CP and lymph nodes but were absent from $\mathrm{MRL} /+\mathrm{CP}$ (Figure 3A, outlined in red; the full list of genes is provided in Supplemental Table 2). Differential expression analysis was performed comparing MRL/lpr relative to MRL/+ CP, yielding a group of 1588 significantly upregulated genes and 83 downregulated genes (Figure 3B). Focusing specifically on the differentially regulated cytokines and chemokines and their receptors between these tissues revealed a signature of lymphoid tissue induction, including secondary and tertiary lymphoid induction signals. We also found a strong type II $(\gamma)$ interferogenic signal, which included expression of Cxcl10, Ccl5, Tnfsf10 (TRAIL), 
Reticulin

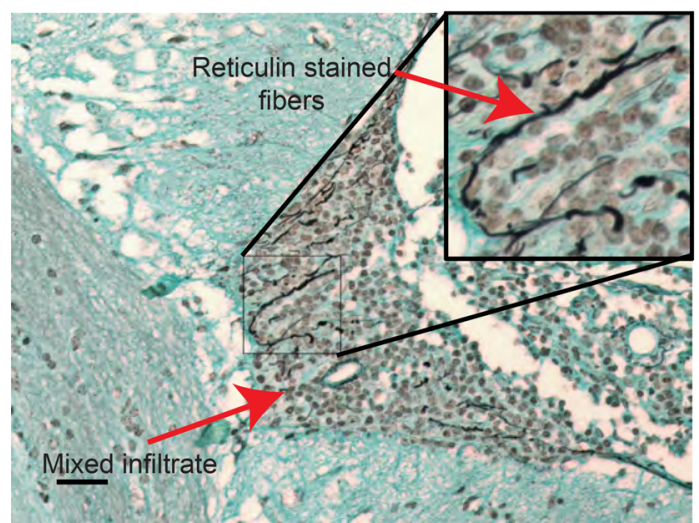

TEM

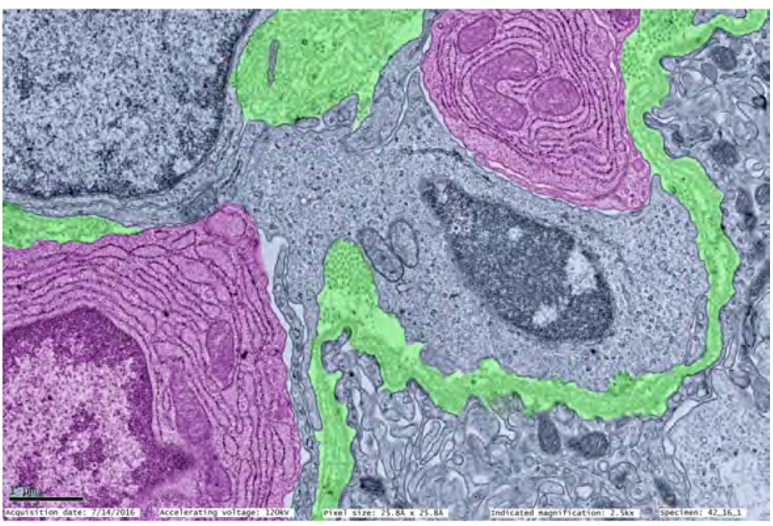

Figure 2. Stromal reorganization occurs within the CP cellular infiltrate. Reticulin staining (left) and TEM (right) reveal organizational structures within the mixed infiltrate consisting of collagen fibers, which appear black in the reticulin stain and are highlighted in green in the TEM image. Plasma cells are apparent as well in the TEM image, highlighted in pink. Scale bars: $50 \mu \mathrm{m}$ (light microscopy), $1 \mu \mathrm{m}$ (electron micrograph). Original magnification, $\times 100$ (inset).

and Casp1 (Figure 3C and Supplemental Table 2) (34). We further evaluated for differential expression of genes associated with posttranslational modification of the constituent proteins of peripheral node addres$\sin$ (PNAd), typically expressed by high endothelial venules and important for the recruitment of naive and certain memory lymphocytes $(35,36)$. We found upregulation of several PNAd components, including Chst3, Cmah, and St8sia4 (Supplemental Figure 1G), which was supported by positive PNAd staining (Supplemental Figure 1E). Selected genes differentially expressed between MRL/lpr and MRL/+ CP, including $L t b, C x c l 3$, Ifng, and $C c r 7$, were validated by reverse transcription quantitative PCR (RT-qPCR) (Figure 4). Interestingly, when evaluating 8-week-old MRL/lpr CP for expression of certain genes revealed by RNASeq, we found significant elevation of several cytokines and chemokines, including $L t b$ and Cxcl10, before there was any significant lymphocytic infiltration (Figure 4). Finally, Gene Ontology (GO) assessment identified MRL/lpr CP expression signatures associated with lymphocyte migration and chemotaxis lymphocyte migration (GO:0072676, $P=1.9 \mathrm{E} 10^{-8}$ ), lymphocyte chemotaxis (GO:0048247, $P=1.02 \mathrm{E} 10^{-5}$ ), and regulation of lymphocyte migration (GO:2000401, $\left.P=1.12 \mathrm{E} 10^{-8}\right)$ - further supporting concerted cell recruitment and organization in this tissue.

The CP infiltrate is organized according to chemokine gradients and intercellular interactions. We examined the MRL/lpr CP for CXCL13, a chemokine integral to lymphoid organization, and found extensive CXCL13 deposition localized in regions rich with B cells and follicular dendritic cells (Figure 5A). We similarly evaluated the $\mathrm{CP}$ cellular infiltrates for evidence of antigen presentation, finding both IBA1/CD4 interactions (Figure 5B) as well as MHCII/CD3 colocalization (Figure 5C), consistent with antigen presentation to $\mathrm{T}$ cells. Interactions between antigen-presenting cells and $\mathrm{T}$ cells were prominently apparent by scanning electron microscopy (SEM) as well (Figure 5D). Additionally, GO term analysis of MRL/lpr CP transcripts revealed significant enrichment for gene expression related to antigen presentation, the immunological synapse, and lymphocyte activation and stimulation (e.g., Lck, Zap70, Icos, Tap2, Cd28, and Cd80), further identifying $\mathrm{T}$ cell activation within the lymphoid structures (Figure 6).

$C P$-infiltrating lymphocytes are phenotypically characteristic of lymphoid follicles with GC activity. After determining that the nature and organization of the cells infiltrating the CNS of mice with lupus were consistent with lymphoid aggregates generated in response to specific lymphoid tissue-inducing mediators, we characterized individual cell phenotype markers that define both the lymphoid follicle and GC reaction. Again, GO analysis provided valuable insight, identifying multiple stages of $\mathrm{B}$ cell maturation and differentiation as evidenced by enhanced transcription of various genes related to $\mathrm{B}$ cell maturation and GC activity, such as Klhl6, Ada, Tnfrsf13c (BAFF receptor), Tnfsf13b (BAFF), and Cdh17 (Figure $7 \mathrm{~A})$. Within the $\mathrm{CD} 4^{+} \mathrm{T}$ cell population, we identified expression of the transcription factor BCL6, which drives Tfh cell responses and is essential in the formation of GC with active somatic hypermutation (Supplemental Figure 1F). Additionally, local proliferation of $\mathrm{CD} 4^{+}$cells was evident by PCNA costaining (Supplemental Figure 1B), suggesting in situ activation. Upon further characterization of the $\mathrm{B}$ cell population, we found $\mathrm{B}^{2} 20^{+} / \mathrm{IgM}^{+}$cells (transitional B cells pending activation; Figure $7 \mathrm{~B}$ ), as 

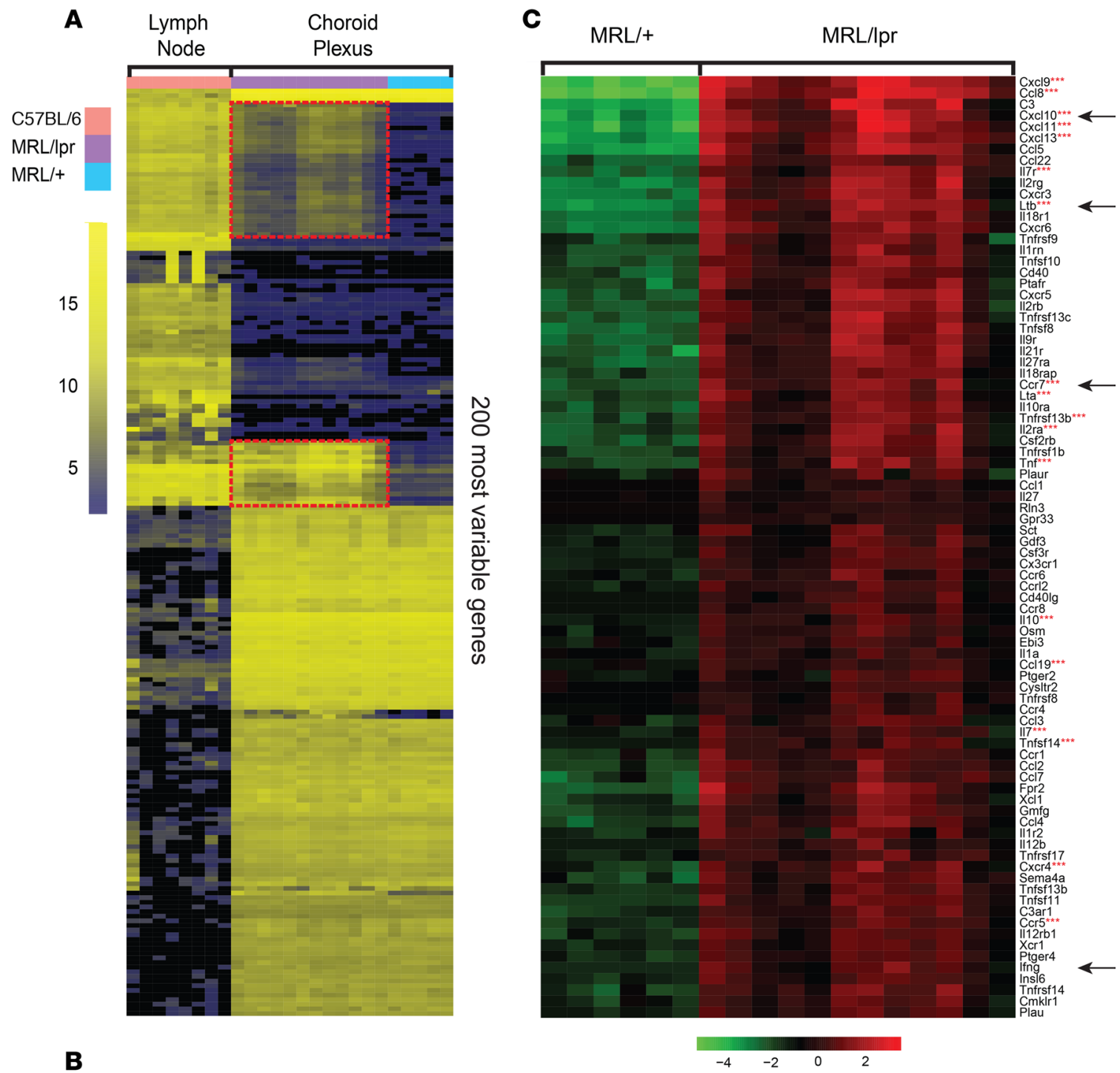

B

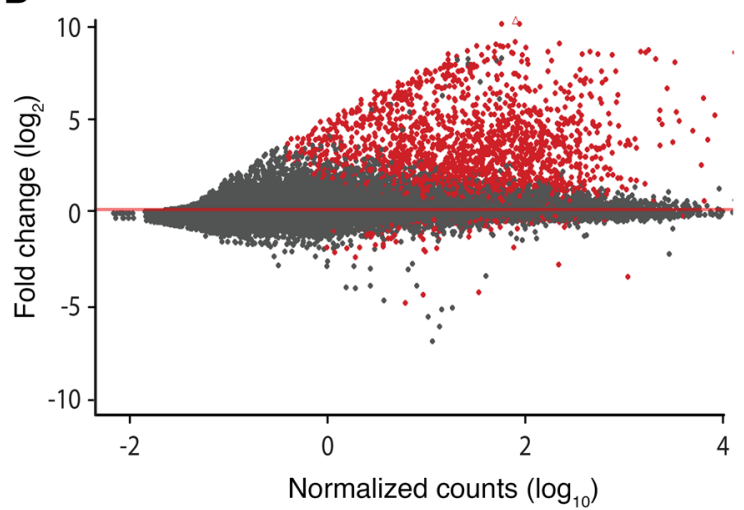

Figure 3. Enhanced expression of lymphoid tissue inducing molecules in lupus CP. (A) Evaluation of the 200 most variable genes between B6 lymph nodes, control MRL/+ CP, and MRL/Ipr CP revealed several distinct sets of transcripts, including sets of genes with high expression in lymph node and $\mathrm{MRL} / \mathrm{lpr}$ CP but not in MRL/+ CP (outlined in red). (B) Comparing MRL/+ and MRL/Ipr transcriptomes revealed 1671 variably expressed genes (red dots), including many cytokines and chemokines and their receptors. (C) When focusing on diverging immunological transcripts between MRL/+ and MRL/ Ipr CPs, multiple genes associated with secondary lymphoid organs and TLS formation are highly expressed (denoted by 3 red asterisks). Arrows point to genes evaluated by RT-qPCR. 


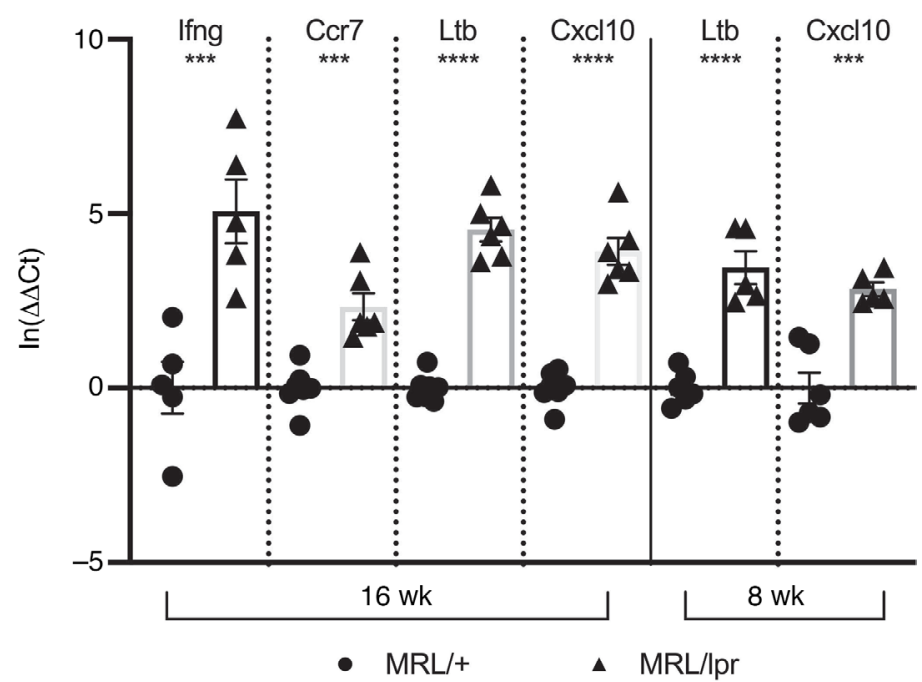

Figure 4. RNA-Seq confirmed by RT-qPCR with early evidence of TLS formation. Selected genes, identified by arrows in Figure 3C, were evaluated by RT-qPCR at 8 and 16 weeks of age. Evaluation at 8 weeks of age revealed high expression of genes related to $\mathrm{CC}$ formation $(L t b)$ and IFN- $\gamma$ activity $(C x c / 10)$. Mean \pm standard error of the mean values are shown; $n=5$. Two-tailed Student's $t$ test. ${ }^{* *} P<0.001 ;{ }^{* * *} P<0.0001$.

well as B220 $/ \mathrm{GL7}^{+}$(activated/GC B cells; Supplemental Figure 1C), GL7 ${ }^{+} / \mathrm{AID}^{+}$(GC B cells undergoing class switch recombination and somatic hypermutation; Figure $7 \mathrm{C}$ ), and $\mathrm{B} 220^{+} / \mathrm{PCNA}^{+}$cells (B cell proliferation; Supplemental Figure 1D), all subsets typically found in lymphoid follicles. Additionally, there were localized clusters of $\mathrm{SDC1}^{+}\left(\mathrm{CD} 138^{+}\right)$plasma cells, with the majority positive for cytosolic IgG (Figure 7D).

Ultrastructural evaluation of the lupus $C P$ revealed extensive inflammatory pathology and $T$ cell dissemination to the CNS across the CP epithelium. Further evaluation of the CP was performed by electron microscopy because this modality allows for direct evaluation of intercellular interactions as well as subcellular features of the tissue, including cell localization. TEM revealed that lymphocytes were often found engulfed by CP epithelial cells and localized within their membrane borders (Figure 8A), with no apparent disruption of interepithelial tight junctions (Figure 9B), which together are strongly suggestive of transepithelial T cell migration, a finding that we recently described more extensively elsewhere (37). SEM demonstrated a normal-appearing $\mathrm{CP}$ in MRL/+ mice (Figure 8B), whereas there was extensive leukocyte infiltration and stromal expansion in the MRL/lpr mice (Figure 8C). TEM further confirmed the presence of clusters of leukocytes and plasma cells within the CP stroma (Figure 9A). Evaluation of the villous processes of the $\mathrm{CP}$ by TEM revealed several striking inflammatory features. Most remarkable was the gross distortion of the epithelial basement membrane, including extensive thickening and deposition of a granular, osmophilic substance (Figure 9C). This finding is consistent with antibody or immune complex deposition, much as described in the CP of patients with SLE $(16,38)$. As mentioned above, intercellular junctional complexes were intact, suggesting that crossing of any cell or substance into the cerebrospinal fluid (CSF) space would occur in a transcellular fashion.

Leukocyte infiltration occurs through the CP of SLE patients with neuropsychiatric disease. We screened the Montefiore Medical Center (Bronx, New York) patient database for those individuals with a diagnosis of SLE who underwent brain assessment at autopsy. Archival tissue including the $\mathrm{CP}$ of the lateral and fourth ventricles was sampled and stained for the pan-leukocyte marker CD45. Patients $(n=12)$ had a median age of $45.5 \pm 15.9$ at the time of autopsy; $75 \%$ were female, $40 \%$ were African-American, and $60 \%$ were Hispanic. At the time of death, only 2 patients had evidence of significant cerebrovascular disease, whereas the majority had other neuropsychiatric conditions, including depression, chronic headache, seizure disorder, altered mental status, and psychosis. Two were found to have intrathecal fungal disease and were consequently excluded from histological analysis. We found that the $\mathrm{CP}$ served as a site for leukocyte migration into the CNS in several patients with lupus, including instances of larger groupings of lymphocytes (Figure 10). Interestingly, when evaluating leptomeningeal structures and associated perivascular Virchow-Robin spaces, we routinely found substantial numbers of lymphocytes, as well as cells penetrating into adjacent parenchyma (data not shown). 
A

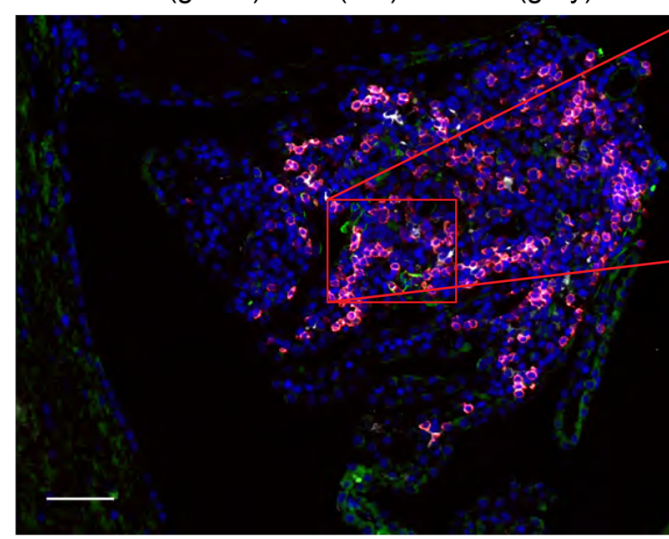

B

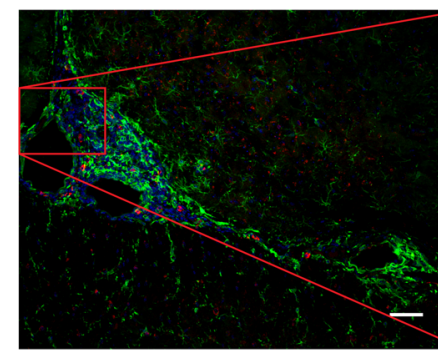

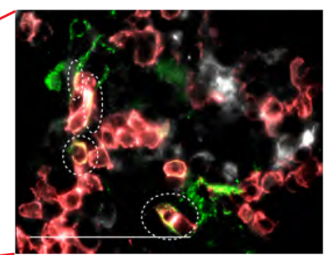

CD21/B220

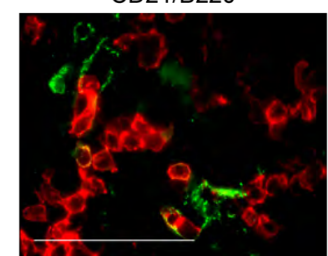

D

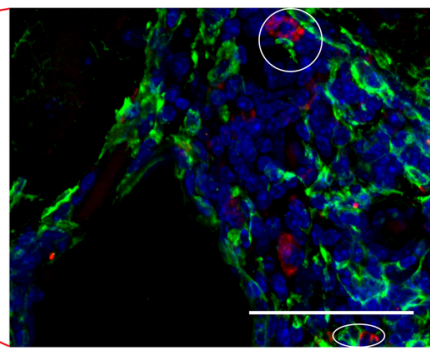

CD3(green)/MHCII(red)

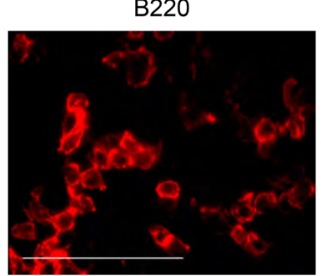

CXCL13

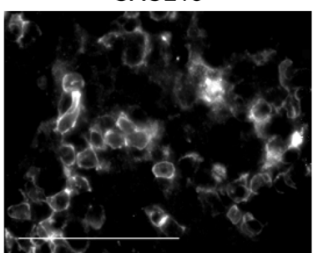

SEM

C
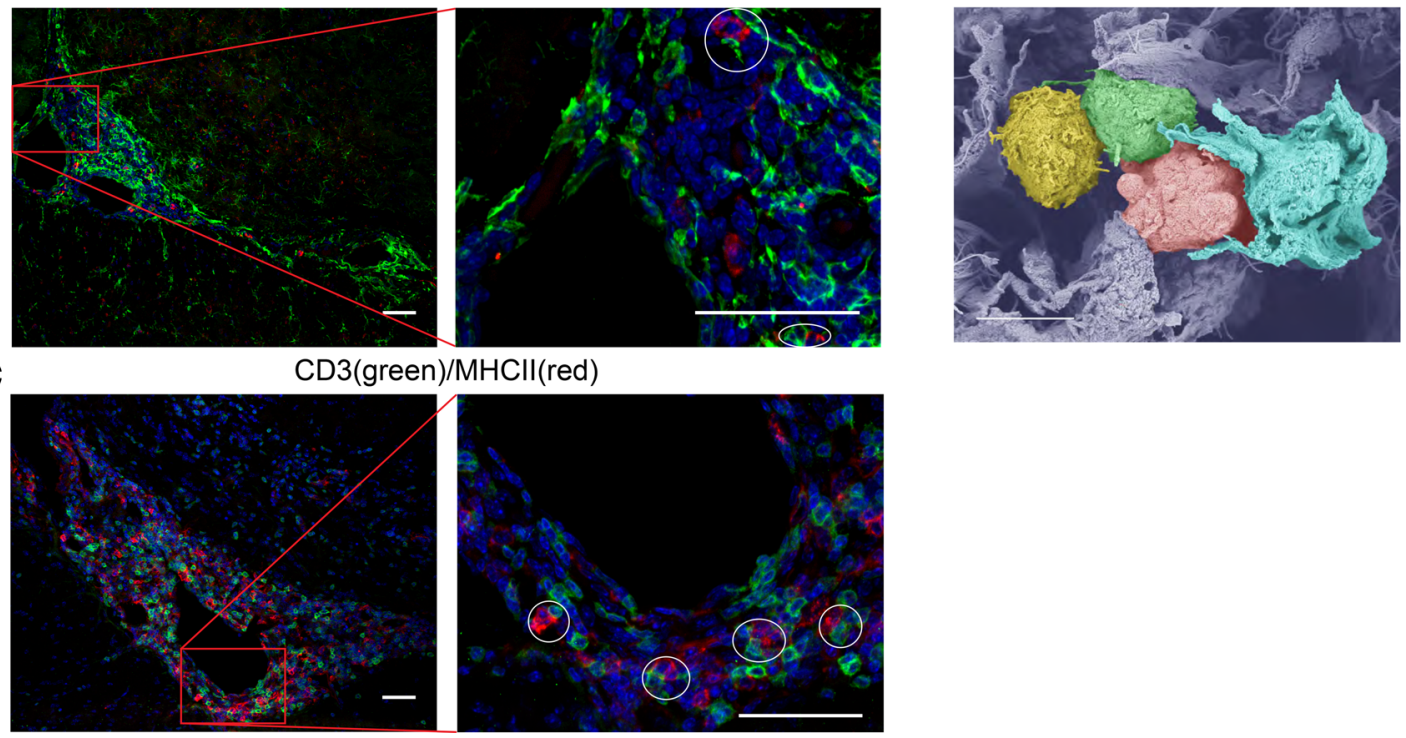

Figure 5. MRL/Ipr CP tissue displays chemokine gradients and antigen presentation. (A) Staining for FDC (CD21), B cells (B220), and CXCL13 revealed B cell alignment with FDC processes as well as CXCL13, the cytokine responsible for colocalization of these cells. (B) CD4+ ${ }^{+}$cells were found juxtaposed to IBA1+ monocytes, as were (C) CD3 and MHCII. (D) Pseudocolored SEM revealed multiple cell types in close interaction, including dendritiform cells (shown in light blue) with lymphocytes (shown in green and pink), which appear to be further communicating with one another (represented as green and yellow cells). Scale bars: $50 \mu \mathrm{m}$ (A-C), $5 \mu \mathrm{m}$ (D).

\section{Discussion}

Although there have been previous reports identifying several of the cell types present within the $\mathrm{CP}$ of MRL/lpr mice $(20,31)$, there has been very little characterization of the mechanisms through which they infiltrate and the functional consequences of their presence. We found that infiltrating cells in the CP of NPSLE mice generate TLSs, with in situ GC activity, likely in response to an elaborate transcriptional signature that includes typical signaling axes as well as potential involvement of novel pathways. We have additionally found that the $\mathrm{CP}$ epithelium serves as a trafficker of lymphocytes in a concerted transepithelial fashion from subepithelial stroma into the ventricles.

The pathogenesis of NPSLE is complex and multifactorial. Nevertheless, as in the case of other lupus-related pathologies, it is believed that autoantibodies may be important contributors $(39,40)$. However, the relationship between serum autoantibodies and brain pathology in SLE is inconsistent (41-44). Although there has been speculation regarding potential routes of entry for neuropathic autoantibodies into the CNS of patients with SLE (45), there is little direct evidence of blood-brain barrier (BBB) or other blood-CNS barrier disruption (46). This does not, however, preclude production of neuropathic autoantibodies in localized, ectopic lymphoid structures, as has been recently reported in lupus nephritis (22). Furthermore, this could help account for the variable seropositivity of patients with NPSLE as well. 


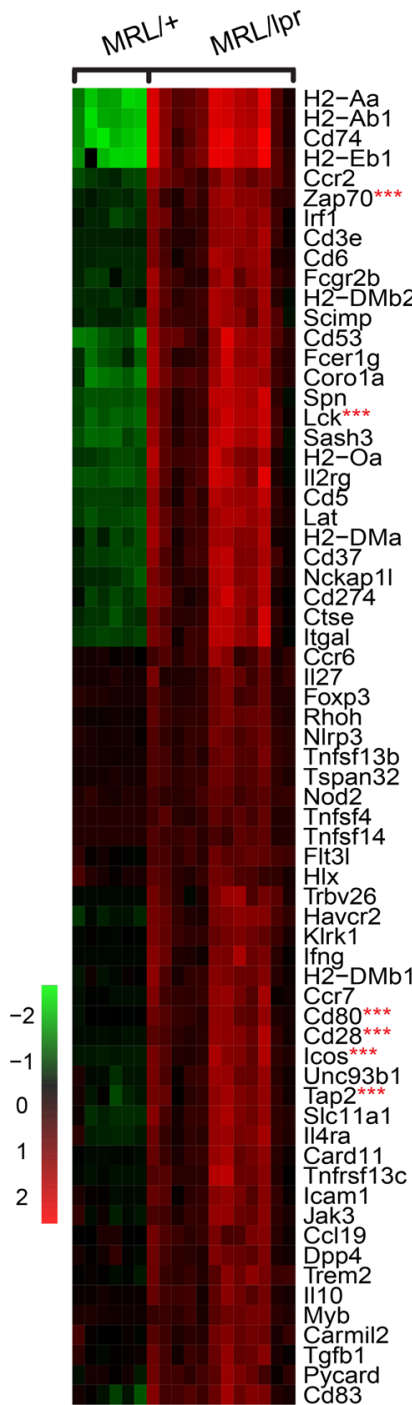

Figure 6. MRL/Ipr CP gene expression profile is consistent with antigen presentation and its effects. Evaluation of 120 distinct genes linked to GO terms associated with T cell activation and antigen presentation (GO:0019886, GO:0002579, GO:0031295, G0:0001772, and G0:0043370), revealed increased expression of 66 genes relating to these processes. Selected critical genes are denoted by 3 red asterisks.

Given the restrictiveness of the $\mathrm{BBB}$, the role of the $\mathrm{CP}$ in mediating neuroimmunopathology has also recently grown in importance. The $\mathrm{CP}$ functions as an important neuroimmune interface under basal physiological conditions $(12,47)$, as well as in inflammation $(14,48)$. Additionally, the $\mathrm{CP}$ has been implicated in human NPSLE, as evidenced by MRI $(18,49)$ as well as anecdotal reports identifying IgG deposition within the CP basement membrane in biopsy samples (16). Furthermore, our recent work evaluating BBB function and routes of immune penetration into the CNS has proved revealing in the model used herein; BBB function in particular as it relates to interendothelial tight junctions appears to be mostly conserved, and the $\mathrm{CP}$ is in fact a conduit for immunoglobulin transmission and lymphocyte migration (37). The various potential modes of immune migration through the CNS in SLE have been recently reviewed, including discussion of the relative roles of the BBB and $\mathrm{CP}$, as well as the potential involvement of dural lymphatics and the glymphatic pathways (46).

Our discovery of lymphoid structures in the $\mathrm{CP}$ is supported by several other recent observations that collectively suggest that the cognitive and emotional deficits found in NPSLE may not rely solely on systemic autoimmunity. Specifically, the onset of NPSLE occurs well before there is a systemic autoimmune burden in MRL/lpr mice (7), and neuropsychiatric manifestations are frequently an early feature of pediatric SLE (9). Moreover, using bone marrow transplantation to arrest autoimmunity in MRL/lpr mice, we found little change in behavioral outcomes despite near reversal of systemic disease (4). Additionally, tissue-specific TLSs have been found in various autoimmune states, allowing for the generation of immune responses to localized antigens (28). This occurs separately from traditional modes of acute leukocytic infiltration resulting from remote antigen presentation and most frequently is found in cases of chronic inflammation, both in mouse models and in humans, as a consequence of stimulation by soluble molecules, including LT (50) and CXCL13 (23). 


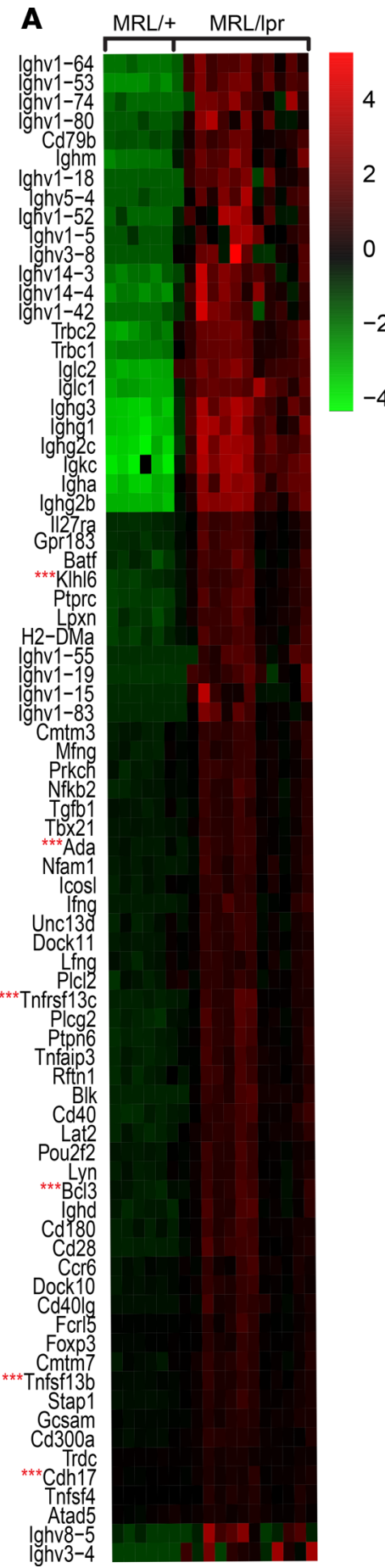

B

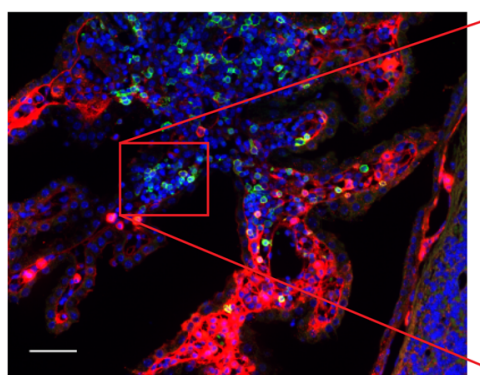

C

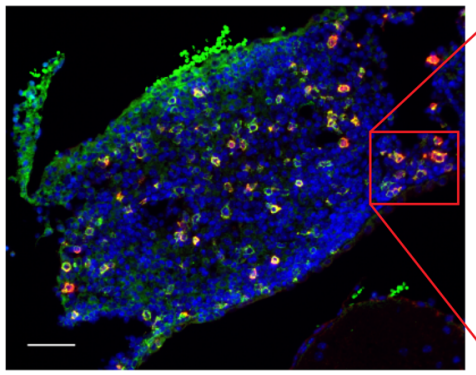

D
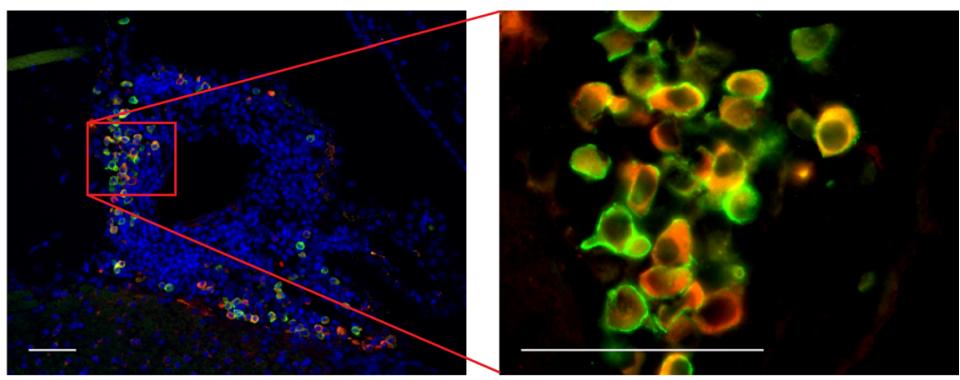

Figure 7. CP-infiltrating cells contain lymphoid follicular subsets and show GC activity. (A) Assessment of 177 distinct genes relating to $B$ cell differentiation, B cell receptor signaling, and GC activity (G0:0002315, G0:0002312, ५0:0050855, G0:0002335, С0:0050853, G0:0002636, and С0:002467) revealed increased expression of 79 genes, including key genes of B cell activation and $\mathrm{GC}$ formation (denoted by 3 red asterisks). (B) B220/IgM costaining cells. (C) GL7/ AID costaining, consistent with GC B cells undergoing class switch recombination and somatic hypermutation. (D) SDC1 (CD138)/IgG double-positive cells are IgG-producing plasma cells. Scale bars: $50 \mu \mathrm{m}$.

Notably, however, robust lymphocytic infiltration and cell-mediated inflammation is not a common finding within the CNS parenchyma of SLE patient tissue examined postmortem. We believe that this is likely a consequence of tissue analysis in patients who have been treated with long-term or at least recent potent cytotoxic medications. Indeed, skin and renal biopsies performed on SLE patients with active disease are routinely positive for mononuclear infiltrates, including previously described TLSs described in the kidney (51). Similarly, when MRL/lpr mice are treated with aggressive immunosuppression with cyclophosphamide (a cytotoxic agent), they experience attenuated behavioral abnormalities and modulation of cellular infiltrates (52).

RNA-Seq proved to be a powerful tool in identifying the complex and multifaceted transcriptional activity that feeds into TLS generation. When evaluating the $\mathrm{CP}$ of diseased MRL/lpr mice, we found marked elevation of many chemotactic factors, including $C x c l-9,-10,-11,-13$, and -19 , as well as $C c l-3,-4,-5,-6,-7,-8,-12,-19$, and -22 . 
A
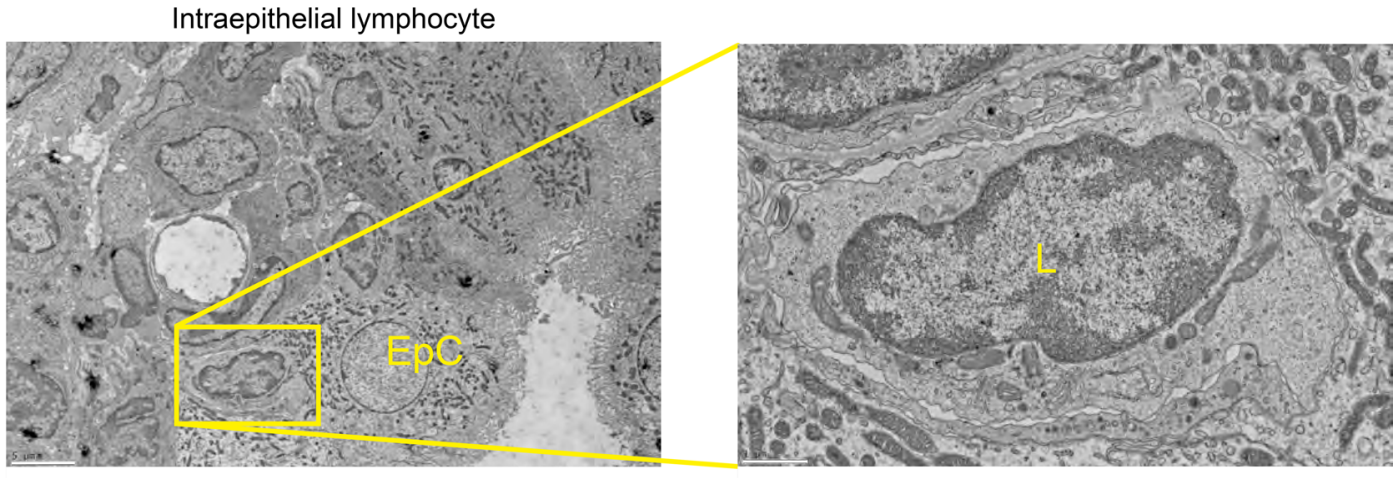

B

$\mathrm{MRL} /+$ third ventricle, SEM

\section{C}

MRL/Ipr third ventricle, SEM
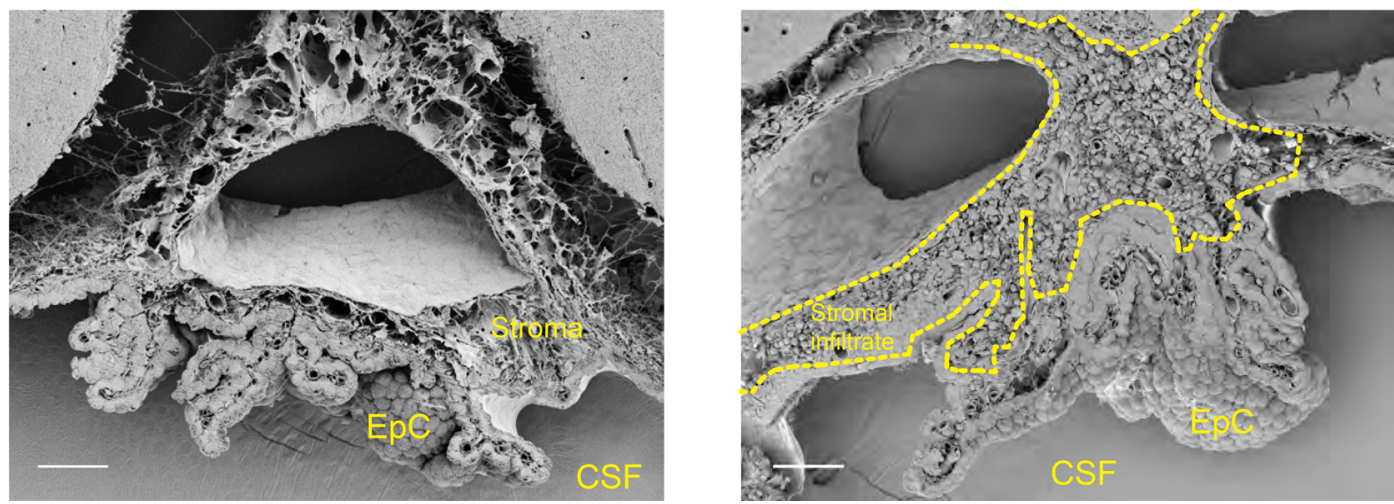

Figure 8. Ultrastructural evaluation reveals stromal expansion and T cell dissemination across the epithelium. (A) Small round cells consistent with lymphocytes were routinely found wholly within epithelial cell membranes. Scale bar: $5 \mu \mathrm{m}$ (left), $1 \mu \mathrm{m}$ (right). EpC, epithelial cell; L, lymphocyte. (B) $\mathrm{MRL} /+$ and (C) MRL/Ipr SEM demonstrating a typically appearing CP architecture in MRL/+ mice and extensive cellular infiltration in MRL/Ipr mice. Scale bars: $5 \mu \mathrm{m}$. CSF, ventricular cerebrospinal fluid space.

Of these, Cxcl13 and Ccl19 are canonical factors in the lymphoid organization of B and T lymphocytes, respectively. Importantly, in the chronic inflammation associated with NPSLE, these chemokines are likely effectors in the recruitment and organization of leukocytes in the formation of a TLS. Other key molecules in the in situ generation of lymphoid structures mediate LT- $\alpha /-\beta$ signaling, a pathway highly expressed in the transcriptomic signature of the lupus CP. Interestingly, when evaluating the $\mathrm{CP}$ of young MRL/lpr mice well before any significant lymphocytic infiltration, we were surprised to find robust expression of Ltb, Ifng, Tnfa, Ccl5, and Cxcl10, indicating that the process of TLS generation is likely driven by CP epithelial- or even parenchyma-derived signaling. In future studies, it will be interesting to parse out these early, initiating events in this process.

It is important to point out some potential limitations of the bulk RNA-Seq approach, including that enrichment of specific genes may be a function of the number of infiltrating cells and that the expression of genes related to GC responses in CP-infiltrating immune cells may be further appreciated by a comparison to immune cells isolated from unaffected tissues. Nevertheless, we did include data from lymph nodes. Moreover, the bulk RNA-Seq results were supplemented and supported by the comprehensive immunofluorescence studies. Using single-cell sequencing techniques in future studies, along with analytical tools that allow for determination of $\mathrm{T}$ cell and $\mathrm{B}$ cell receptor clonality at the single-cell level, will facilitate a closer look at these and related questions. Similarly, direct evaluation of the pathogenicity of B cell clones or their antibody products through adoptive transfer studies would be valuable.

When evaluating the cellular phenotypes of the CP infiltrate, we found many features that strongly supported the presence of TLSs. The structural features of lymphoid organs were apparent, including reticular fibers and collagen bundles and distinct $\mathrm{B}$ and $\mathrm{T}$ cell domains. We found that $\mathrm{B}$ cells were clustered according to chemokine gradients and were regionally colocalized with $\mathrm{CD} 21^{+}$follicular dendritic cells, as is expected for antigen presentation to B cells. Similarly, T cells were regionally colocalized with IBA $1^{+}$cells and had membrane colocalization with MHCII on neighboring cells. We further found cells expressing markers characteristic of various stages of B cell development, including 
A

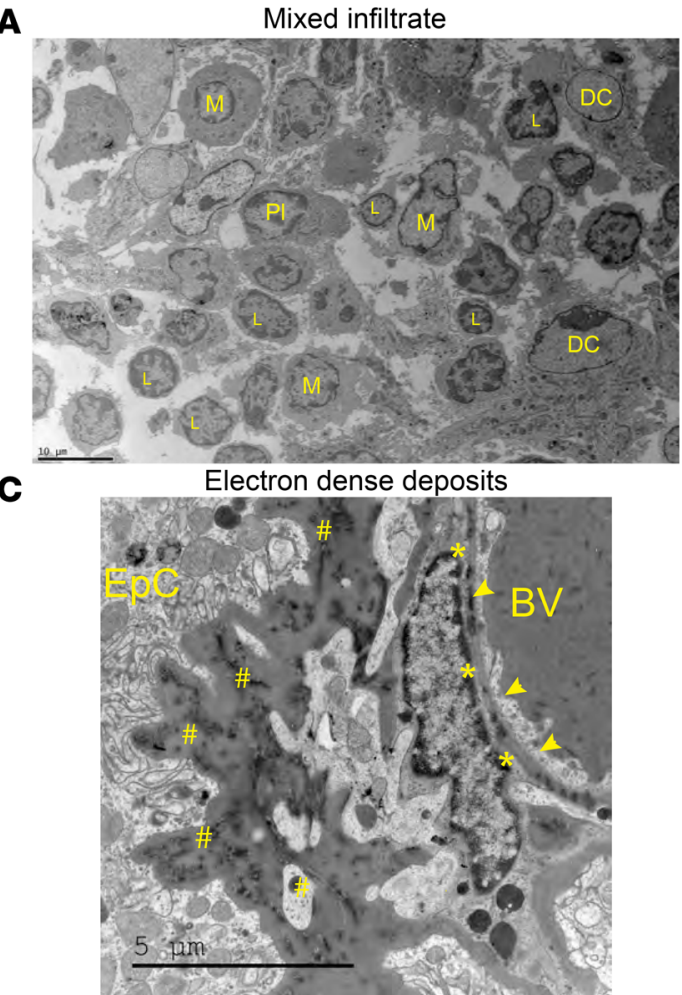

B

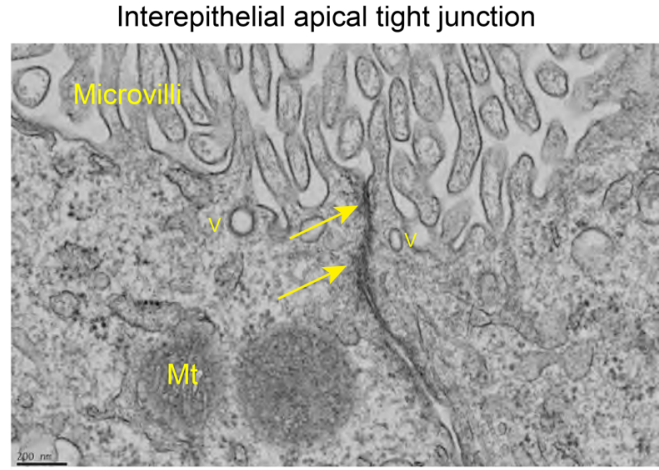

Figure 9. Ultrastructural evaluation reveals extensive leukocyte aggregation and inflammatory changes to the stroma, while maintaining tight junction integrity. (A) Various cell types were readily identifiable by TEM, including lymphocytes, monocytes, dendritic cells, and plasma cells, confirming the immunofluorescence findings. Scale bar: $10 \mu \mathrm{m}$. M, macrophage; PI, plasma cell. (B) Interepithelial tight junctions found near the apical surface of the CP cells appear normal. Scale bar: $200 \mathrm{~nm}$. Arrows, tight junction; V, vesicle; Mt, mitochondria. (C) The CP stromal space has extensive thickening of the basement membrane and deposition of osmophilic substances, consistent with immune complexes or antibodies. Scale bar: $5 \mu \mathrm{m}$. BV blood vessel; arrowheads, endothelial cells; *, endothelial basement membrane deposition; \#, epithelial basement membrane deposition.

transitional, activated, and GC B cells, along with activated CD4+ and Tfh cells. GC activity was supported by RNA-Seq as well, with several genes, including Klhl6 (B cell receptor signal transduction), Ada (GC B cell survival), Tnfrsf13c (BAFF receptor), and Tnfsf13b (BAFF), showing high transcript levels $(53,54)$. Finally, evidence of lymphocyte migration through the $\mathrm{CP}$ appears to be a feature of NPSLE in patients as well. Interestingly, in addition to those found in the CP, we routinely found $\mathrm{CD} 45^{+}$cells in the pia-lined perivascular space of penetrating vessels (aka Virchow-Robin space). This potential space, which is anatomically contiguous with the ventricles, has been found to house the aforementioned glymphatic system. It is tempting to speculate that the CP stroma functions as a leukocyte conduit, fed by the fenestrated choroidal vasculature and the hyperpermissive epithelium, rather than through the highly impermeable parenchymal BBB vessels.

To identify the role of CXCL13, an essential chemokine for the recruitment of naive B cells to TLSs, we chose to use staining protocols to maximize the identification of the secreted, interstitial CXCL13 signal. Consequently, the pattern of staining we identified demonstrated CXCL13 on the surface of B220+ (B cells), which would express CXCR5 (the receptor for CXCL13). Moreover, CD21 ${ }^{+}$cells consist of an extensive network of processes extending from a small number of cell bodies, which would not typically be evident in the thin-section microscopy we used, whether in primary or secondary lymphoid tissue or in the TLSs demonstrated here. Finally, because we are describing an atypical lymphoid structure occurring in a tissue not previously described to have this capacity, staining patterns may not be identical to those of primary or secondary lymphoid organs.

Further supporting the identification of the mixed lymphoid aggregates in the CP of MRL/lpr as TLSs are our recent studies of the $\mathrm{T}$ cell population (55). We found that a subset of $\mathrm{CP}$-infiltrating $\mathrm{CD} 4^{+} \mathrm{T}$ cells in MRL/lpr mice displayed a Tfh-like phenotype, as evidenced by their surface markers and IL-21 expression. Moreover, flow cytometric analysis confirmed the significant expression of IFN- $\gamma$ and BCL6 in these cells, consistent with the RNA-Seq data described in the current paper. Importantly, while T cell infiltration is also present in other organs in the MRL/lpr strain (e.g., lung), those infiltrates include a significant number of double-negative T cells (56). Double-negative T cells, however, were not increased in the CP (Figure 1 and ref. 55), further supporting a specific immunological process involving structure and organization in this unique tissue, rather than passive lymphocytic accumulation.

Future studies to conclusively confirm the pathogenicity of the CP-localized TLSs that develop in parallel with worsening behavioral disease, including their disruption by various pharmacological or genetic means, will 

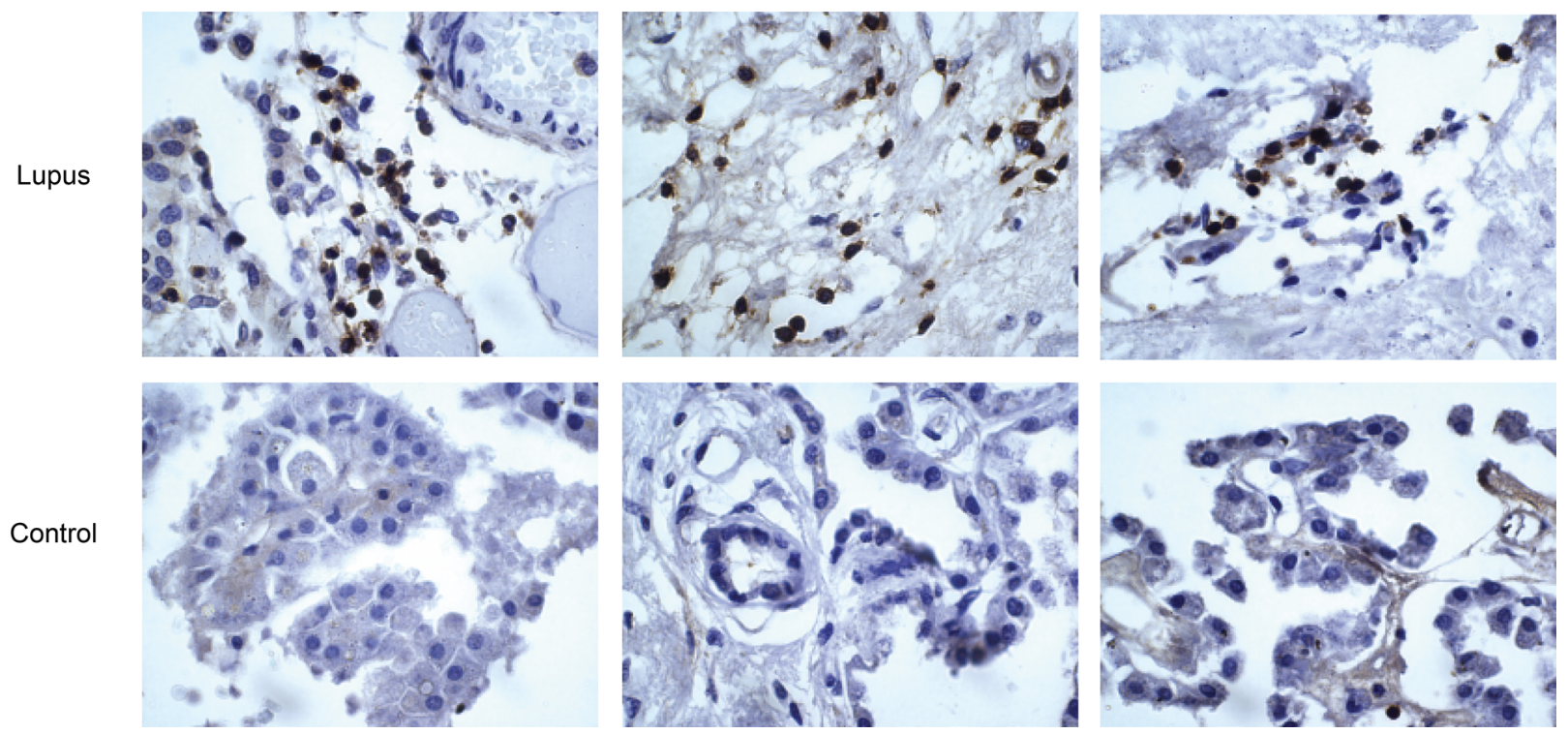

Figure 10. Human CP tissue contains leukocytic infiltration. IHC staining revealed clusters of CD45+ cells with the morphological characteristics of lymphocytes in the CP stroma of patients with SLE but not in controls.

be an important next step. Nevertheless, we have previously demonstrated that knock out of Fn14 (TNFSF12 receptor) decreases the extent of $\mathrm{CP}$ lymphocyte infiltration, with concomitant improvement in behavioral outcomes (21). Interestingly, TLS formation has been recently linked to the pathogenesis of lupus nephritis, a major contributor to SLE-related mortality. Moreover, several studies have evaluated mice with lupus and human lupus tissue, finding that BAFF (51) and CXCL13 (57) are contributors to TLS formation in the kidney. Furthermore, clonotypic plasma cell responses with in situ somatic hypermutation have been demonstrated in human lupus kidney biopsies (22). It is interesting to speculate whether any of the beneficial effects of BAFF inhibition in human lupus are mediated through perturbation of TLS formation.

The CNS is quite unique immunologically and is likely not as susceptible to the formation of lymphoid aggregates as other tissue types, such as the synovium (23), kidney (22), and large vessels (50). The blood-brain and blood-CSF barriers tightly regulate movement of both cellular and soluble immune effectors into the CNS. Even in the case of multiple sclerosis, ectopic lymphoid follicles were not found within CNS parenchyma, but rather in the meninges (58), which have been recently identified as a region rich in lymphatics with potent immunosurveillant functions (11). Lymphatic vasculature, however, is not thought to be present within brain tissue, which is reliant on the glymphatic system for clearance of interstitial fluid and soluble material (10). We should point out that with rare exceptions tissue sampling is not a routine component of the diagnosis and management of chronic CNS disease processes, including neuropsychiatric lupus, thus limiting the ability to monitor progressive immune activity in the clinical realm as well as quickly perform parallel studies to those performed here in human lupus. Although we did find evidence of lymphocytic clustering in the region of the CP in several patients with NPSLE, which suggests an important translational link to our murine lupus studies, definitive conclusions await the study of additional patients. An alternative approach, which we and others are actively pursuing, is the use of advanced radiographic tools to noninvasively track immune activity at the high resolution required for this purpose.

Localized deep within the CNS and the major producer of its fluid phase, the $\mathrm{CP}$ is uniquely positioned to fulfill many immunoregulatory roles. Importantly, as opposed to the endothelial barrier in the majority of the CNS, the $\mathrm{CP}$ vasculature is fenestrated, with the epithelium providing barrier and selective transport functions. Additionally, beyond its importance in production of CSF, the CP epithelium can direct cellular traffic by localized chemokine secretion. Finally, the vasculature of the $\mathrm{CP}$ is indeed a sprawling plexus, allowing for rapid transmission and dissemination of cells and soluble molecules throughout the subepithelial stroma. Collectively, these features not only allow for effective immune interfacing but also are likely an essential component in the development of the lymphoid structures we identified in NPSLE. 
TLSs may present with high endothelial venules and a new lymphatic supply $(50,59)$, in a process akin to neovascularization that occurs in tumor growth. Because a lymphatic system associated with this TLS has yet to be conclusively identified, our results suggest that the $\mathrm{CP}$ and its fenestrated capillary plexus and basal immunosurveillant functions may be uniquely able to facilitate leukocyte migration and homing. Furthermore, while we found elevated Glycam1 expression (a component of the PNAd complex), immunofluorescence demonstrated a cellular (but not vascular) pattern of PNAd expression interspersed throughout the infiltrate (Supplemental Figure 1E). This finding, when coupled with high expression of glycosyltransferase transcripts, including Cmah, Chst3, St8sia4, and St4gal6, strongly suggests that PNAd substituent proteins are undergoing local posttranslational modification (60) and that leukocyte trafficking into the TLS may be directed by elements other than the vasculature, which is what typically occurs in secondary lymphoid organs.

Our findings provide for an important contribution to the evolving understanding of the $\mathrm{CP}$ as a neuroimmune interface, namely that CP epithelium is capable of supporting lymphoid follicle generation in situ. These structures, which contain the hallmark transcriptional signatures and cells of the GC, were generated in response to a complex expression pattern of critical signaling molecules and may represent yet another important mechanism by which the CP interacts with the immune system, particularly in cases of chronic neuroimmunological disease. Future studies will be directed at determining the pathogenicity of autoreactive antibodies produced in situ, as well as chemotactic factors involved in leukocyte recruitment in patients with NPSLE. Together, these future studies may identify potential therapeutics for managing the neuropsychiatric complications of SLE.

\section{Methods}

Animals. Female MRL/MpJ-Fas ${ }^{\mathrm{pr} / \mathrm{pr}}(\mathrm{MRL} / \mathrm{lpr})$ and MRL/MpJ-Fas ${ }^{+/+}(\mathrm{MRL} /+)$ mice were purchased from the Jackson Laboratory at $5-8$ weeks of age and housed at $21^{\circ} \mathrm{C}-23^{\circ} \mathrm{C}$ on a 12 -hour light/12-hour dark cycle. All animal protocols were approved by the Albert Einstein College of Medicine Institutional Animal Care and Use Committee.

Tissue preparation. Age-matched MRL/lpr and control MRL/+ mice were sacrificed between 5 and 16 weeks of age, representing the time frame from well before CP cellular infiltration through widespread infiltration in the majority of MRL/lpr mice. Mice were perfused with PBS (Corning) before brain isolation and subsequent tissue processing. The $\mathrm{CP}$ from all ventricles was isolated and flash-frozen in liquid nitrogen for subsequent RNA isolation from several cohorts of 4-6 mice. Additional mice at $5(n=5), 8-9$ ( $n$ $=9)$, and 16-17 $(n=12)$ weeks of age were perfused with PBS followed by $2.5 \%$ paraformaldehyde (PFA; MilliporeSigma) and postfixed in $2.5 \%$ PFA at $4^{\circ} \mathrm{C}$ followed by processing for paraffin sectioning.

Archival autopsy tissue from patients with NPSLE was identified from within the Montefiore Medical Center patient database. Exclusion criteria included active CNS infection at time of death, history of intrathecal neoplasm, and prolonged immunosuppression. In particular, younger patients with NPSLE were identified to minimize confounding by long-standing disease and age-related degenerative or neurovascular complications.

RNA isolation and downstream analysis. RNA isolation from CP tissue was performed with the Zymo Research Micro Prep Kit. For RT-qPCR, RNA concentration and quality were evaluated by Nanodrop (Thermo Fisher Scientific). cDNA of total mRNA was generated using the Superscript III First Strand Synthesis kit with poly(A) tail primers (Life Technologies). RT-qPCR was performed in triplicate as described elsewhere (21). For RNA-Seq, RNA yield and quality were evaluated using a Bioanalyzer RNA 6000 Nano Chip (Agilent). RNA samples from the CP of 12- to 16-week-old MRL/lpr $(n=12)$ and MRL/+ $(n=6)$ mice were multiplexed and sequenced over 2 lanes for single-end 100-bp reads on a HiSeq2500 instrument (Illumina) using the TruSeq Library Prep Kit v2 (Illumina). The RNA-Seq data described below have been deposited in Gene Expression Omnibus (GEO) (61) and are accessible through accession GSE99030 (https://www.ncbi.nlm.nih.gov/geo/query/acc.cgi?token=cvqpooeorvojran\&acc=GSE99030).

Publicly available RNA-Seq data for unmanipulated 12-week-old C57BL/6J lymphoid tissue were obtained from the National Center for Biotechnology Information's GEO using data set numbers GSM1533040-1, GSM1533051-3, and GSM1533060-2 (47). Demultiplexed FASTQ files were aligned to the mouse reference genome GRCm38 using STAR aligner (version 2.5.3a) (62). The STAR reference index was created using a transcript annotation in a general transfer format (GTF) file from Ensembl release 88 . Uniquely mapped reads to the reference genome were counted using featureCounts (version 1.26.0) (63) and the Ensembl 88 GTF by collapsing on the gene level. For all differentially expressed genes, data are represented as natural log-transformed transcripts per million with row means subtracted. Gene ontology was evaluated using the GO Consortium annotation and ontology tool kit (64). 
Immunostaining. PFA-fixed tissue was paraffin embedded and $5-\mu \mathrm{m}$ sections were used for the majority of stains, with the exception of CD3/MHCII and CD4/IBA1 staining, which were performed on 7 - to $10-\mu \mathrm{m}$ frozen sections (without the need for antigen retrieval). Fluorescent staining was performed as described elsewhere (4); antibodies, concentrations, and antigen retrieval conditions are provided in Supplemental Table 1. Tissue was treated with minimal detergents to prevent washout of interstitial and cell surface chemokine deposits. For all stains, control MRL/+ mice were included and primary antibodies were routinely withheld to control for nonspecific secondary staining. Chromogenic staining of human tissue was performed similarly, with the additional use of DAB and hematoxylin counterstaining (VWR). All secondary antibodies were purchased from Jackson Immunoresearch. Slides were visualized using a Thermo Fisher Scientific Evos FL Auto2. Secondary antibody controls were used for all staining, and image acquisition was adjusted to show signals only above the background defined by secondary antibody control staining.

Electron microscopy. For TEM, brains were dissected and fixed as described previously (65), then examined under a JEOL 1400Plus electron microscope at $80 \mathrm{kV}$. Samples for SEM were prepared as described previously (66) and examined under a Zeiss Supra Field Emission Scanning Electron Microscope, using an accelerating voltage of $2 \mathrm{kV}$.

Statistics. Statistical evaluation and graphing of RT-qPCR were performed using GraphPad Prism. CP RT-qPCR data were calculated using the $\triangle \Delta \mathrm{Ct}$ method, normalized to expression of Ppia, and logarithmically scaled. Significant differences between groups were calculated by analysis with a 2-tailed Student's $t$ test. Statistical significance was defined as a $P$ value of less than 0.05 . For RNA-Seq, differential expression analysis was performed using DESeq2 (version 1.16.1) (67) and R (version 3.3.3), as described previously $(68,69)$.

Study approval. All animal studies were approved by the Albert Einstein College of Medicine Institutional Animal Care and Use Committee. Use of postmortem human tissue was approved by the Albert Einstein College of Medicine/Montefiore Medical Center IRB.

\section{Author contributions}

ADS designed parts of this study and performed the majority of the experiments. ED performed bioinformatic analysis of sequencing data. SG and MH performed certain histological evaluations. ABZ provided general input as well as particular expertise in the areas of electron microscopy and neurovascular biology. $\mathrm{CP}$ conceived of the project and designed the experimental plan. ADS and CP wrote this manuscript, with $\mathrm{ED}, \mathrm{SG}, \mathrm{MH}, \mathrm{KW}$, and $\mathrm{ABZ}$ reviewing and contributing to the writing.

\section{Acknowledgments}

These studies were supported by training grant T32-GM007288 to AS from the NIH, research grants from the Abisch-Frenkel Foundation (15/H1); the Leona M. and Harry B. Helmsley Charitable Trust (2015PG-ISL007); and the Israel Science Foundation (grant 1882/16) to ABZ and a grant from the National Institute of Arthritis and Musculoskeletal Diseases (R01 AR065594) to CP.

Address correspondence to: Chaim Putterman, Division of Rheumatology, F701N, Albert Einstein College of Medicine, 1300 Morris Park Ave., Bronx, New York 10461, USA. Phone: 718.430.4266; Email: chaim.putterman@einstein.yu.edu.

1. Wallace D, Hahn B. The pathogenesis of SLE. Dubois' Lupus Erythematosus and Related Syndromes. 8th ed. Philadelphia, Pennsylvania, USA: WB Saunders; 2013:25-34.

2. Yoshio T, Hirata D, Onda K, Nara H, Minota S. Antiribosomal P protein antibodies in cerebrospinal fluid are associated with neuropsychiatric systemic lupus erythematosus. J Rheumatol. 2005;32(1):34-39.

3. Steup-Beekman G, Steens S, van Buchem M, Huizinga T. Anti-NMDA receptor autoantibodies in patients with systemic lupus erythematosus and their first-degree relatives. Lupus. 2007;16(5):329-334.

4. Stock AD, Wen J, Doerner J, Herlitz LC, Gulinello M, Putterman C. Neuropsychiatric systemic lupus erythematosus persists despite attenuation of systemic disease in MRL/lpr mice. J Neuroinflammation. 2015;12:205.

5. Jeltsch-David H, Muller S. Neuropsychiatric systemic lupus erythematosus and cognitive dysfunction: the MRL-lpr mouse strain as a model. Autoimmun Rev. 2014;13(9):963-973.

6. Sakić B, Szechtman H, Keffer M, Talangbayan H, Stead R, Denburg JA. A behavioral profile of autoimmune lupus-prone MRL mice. Brain Behav Immun. 1992;6(3):265-285.

7. Gao HX, et al. Depression is an early disease manifestation in lupus-prone MRL/lpr mice. J Neuroimmunol. 2009;207(1-2):45-56. 
8. Marian G, Nica EA, Ionescu BE, Carlogea DG. Depression as an initial feature of systemic lupus erythematosus? A case report. JMed Life. 2010;3(2):183-185.

9. Kohut SA, et al. Depressive symptoms are prevalent in childhood-onset systemic lupus erythematosus (cSLE). Lupus. 2013;22(7):712-720

10. Yang L, et al. Evaluating glymphatic pathway function utilizing clinically relevant intrathecal infusion of CSF tracer. $J$ Transl Med. 2013;11:107.

11. Louveau A, et al. Structural and functional features of central nervous system lymphatic vessels. Nature. 2015;523(7560):337-341.

12. Kunis G, et al. IFN- $\gamma$-dependent activation of the brain's choroid plexus for CNS immune surveillance and repair. Brain. 2013;136(pt 11):3427-3440.

13. Engelhardt B, Wolburg-Buchholz K, Wolburg H. Involvement of the choroid plexus in central nervous system inflammation. Microsc Res Tech. 2001;52(1):112-129.

14. Reboldi A, et al. C-C chemokine receptor 6-regulated entry of TH-17 cells into the CNS through the choroid plexus is required for the initiation of EAE. Nat Immunol. 2009;10(5):514-523.

15. Gershwin ME, Hyman LR, Steinberg AD. The choroid plexus in CNS involvement of systemic lupus erythematosus. $J$ Pediatr 1975;87(4):588-590.

16. Sher JH, Pertschuk LP. Immunoglobulin G deposits in the choroid plexus of a child with systemic lupus erythematosus. $J$ Pediatr. 1974;85(3):385-387.

17. Atkins CJ, Kondon JJ, Quismorio FP, Friou GJ. The choroid plexus in systemic lupus erythematosus. Ann Intern Med. 1972;76(1):65-72.

18. Duprez T, Nzeusseu A, Peeters A, Houssiau FA. Selective involvement of the choroid plexus on cerebral magnetic resonance images: a new radiological sign in patients with systemic lupus erythematosus with neurological symptoms. J Rheumatol. 2001;28(2):387-391

19. Wen J, et al. Neuropsychiatric disease in murine lupus is dependent on the TWEAK/Fn14 pathway. J Autoimmun. 2013;43:44-54.

20. Ma X, Foster J, Sakic B. Distribution and prevalence of leukocyte phenotypes in brains of lupus-prone mice. J Neuroimmunol. 2006;179(1-2):26-36.

21. Wen J, et al. Neuropsychiatric disease in murine lupus is dependent on the TWEAK/Fn14 pathway. J Autoimmun. 2013;43:44-54.

22. Chang A, et al. In situ B cell-mediated immune responses and tubulointerstitial inflammation in human lupus nephritis. $J$ Immunol. 2011;186(3):1849-1860.

23. Manzo A, et al. Systematic microanatomical analysis of CXCL13 and CCL21 in situ production and progressive lymphoid organization in rheumatoid synovitis. Eur J Immunol. 2005;35(5):1347-1359.

24. Kratz A, Campos-Neto A, Hanson MS, Ruddle NH. Chronic inflammation caused by lymphotoxin is lymphoid neogenesis. J Exp Med. 1996;183(4):1461-1472.

25. Kogame T, et al. Possible inducible skin-associated lymphoid tissue (iSALT)-like structures with CXCL13. Br J Dermatol. 2017;177(6):1737-1739.

26. Weiss JM, et al. Novel CXCL13 transgenic mouse: inflammation drives pathogenic effect of CXCL13 in experimental myasthenia gravis. Oncotarget. 2016;7(7):7550-7562.

27. Cyster JG. Chemokines and cell migration in secondary lymphoid organs. Science. 1999;286(5447):2098-2102

28. Pitzalis C, Jones GW, Bombardieri M, Jones SA. Ectopic lymphoid-like structures in infection, cancer and autoimmunity. Nat Rev Immunol. 2014;14(7):447-462.

29. Sakic B, Hanna SE, Millward JM. Behavioral heterogeneity in an animal model of neuropsychiatric lupus. Biol Psychiatry. 2005;57(6):679-687.

30. Chalmers SA, et al. Highly selective inhibition of Bruton's tyrosine kinase attenuates skin and brain disease in murine lupus Arthritis Res Ther. 2018;20(1):10.

31. James WG, Hutchinson P, Bullard DC, Hickey MJ. Cerebral leucocyte infiltration in lupus-prone MRL/MpJ-fas lpr mice roles of intercellular adhesion molecule-1 and P-selectin. Clin Exp Immunol. 2006;144(2):299-308.

32. Igarashi S, Takiguchi M, Kariyone A, Kano K. Phenotypic and functional analyses on T-cell subsets in lymph nodes of MRL/ Mp-lpr/lpr mice. Int Arch Allergy Appl Immunol. 1988;86(3):249-255.

33. Kakkanaiah VN, Nagarkatti M, Bluestone JA, Nagarkatti PS. CD4-CD8- thymocytes from MRL-lpr/lpr mice exhibit abnormal proportions of $\alpha \beta$ - and $\gamma \delta$-TCR ${ }^{+}$cells and demonstrate defective responsiveness when activated through the TCR. Cell Immunol. 1991;137(2):269-282.

34. Zeng W, Miyazato A, Chen G, Kajigaya S, Young NS, Maciejewski JP. Interferon- $\gamma$-induced gene expression in CD34 cells: identification of pathologic cytokine-specific signature profiles. Blood. 2006;107(1):167-175.

35. Lee M, et al. Transcriptional programs of lymphoid tissue capillary and high endothelium reveal control mechanisms for lymphocyte homing. Nat Immunol. 2014;15(10):982-995.

36. Lowe JB. Glycosylation in the control of selectin counter-receptor structure and function. Immunol Rev. 2002;186:19-36.

37. Gelb S, Stock AD, Anzi S, Putterman C, Ben-Zvi A. Mechanisms of neuropsychiatric lupus: The relative roles of the blood-cerebrospinal fluid barrier versus blood-brain barrier. J Autoimmun. 2018;91:34-44.

38. Boyer RS, Sun NC, Verity A, Nies KM, Louie JS. Immunoperoxidase staining of the choroid plexus in systemic lupus erythematosus. J Rheumatol. 1980;7(5):645-650.

39. Ho RC, et al. A meta-analysis of serum and cerebrospinal fluid autoantibodies in neuropsychiatric systemic lupus erythematosus. Autoimmun Rev. 2016;15(2):124-138.

40. Kowal C, et al. Human lupus autoantibodies against NMDA receptors mediate cognitive impairment. Proc Natl Acad Sci U S A 2006;103(52):19854-19859.

41. Rekvig OP, et al. Autoantibodies in lupus: culprits or passive bystanders? Autoimmun Rev. 2012;11(8):596-603

42. Shi ZR, Cao CX, Tan GZ, Wang L. The association of serum anti-ribosomal P antibody with clinical and serological disorders in systemic lupus erythematosus: a systematic review and meta-analysis. Lupus. 2015;24(6):588-596.

43. Lauvsnes MB, Omdal R. Systemic lupus erythematosus, the brain, and anti-NR2 antibodies. J Neurol. 2012;259(4):622-629.

44. Husebye ES, et al. Autoantibodies to a NR2A peptide of the glutamate/NMDA receptor in sera of patients with systemic lupus 
erythematosus. Ann Rheum Dis. 2005;64(8):1210-1213.

45. Huerta PT, Kowal C, DeGiorgio LA, Volpe BT, Diamond B. Immunity and behavior: antibodies alter emotion. Proc Natl Acad Sci U S A. 2006;103(3):678-683.

46. Stock AD, Gelb S, Pasternak O, Ben-Zvi A, Putterman C. The blood brain barrier and neuropsychiatric lupus: new perspectives in light of advances in understanding the neuroimmune interface. Autoimmun Rev. 2017;16(6):612-619.

47. Baruch K, et al. Aging. Aging-induced type I interferon response at the choroid plexus negatively affects brain function. Science. 2014;346(6205):89-93

48. Baruch K, et al. CNS-specific immunity at the choroid plexus shifts toward destructive Th2 inflammation in brain aging. Proc Natl Acad Sci U S A. 2013;110(6):2264-2269.

49. Amaro E, Scheinberg M. Onset of cognitive dysfunction in systemic lupus erythematosus and selective involvement of the choroid plexus. J Rheumatol. 2009;36(11):2554-2555.

50. Grabner R, et al. Lymphotoxin beta receptor signaling promotes tertiary lymphoid organogenesis in the aorta adventitia of aged ApoE $^{-/-}$mice. J Exp Med. 2009;206(1):233-248

51. Kang S, Fedoriw Y, Brenneman EK, Truong YK, Kikly K, Vilen BJ. BAFF induces tertiary lymphoid structures and positions T cells within the glomeruli during lupus nephritis. J Immunol. 2017;198(7):2602-2611.

52. Farrell M, Sakić B, Szechtman H, Denburg JA. Effect of cyclophosphamide on leukocytic infiltration in the brain of MRL/lpr mice. Lupus. 1997;6(3):268-274.

53. Kroll J, et al. The BTB-kelch protein KLHL6 is involved in B-lymphocyte antigen receptor signaling and germinal center formation. Mol Cell Biol. 2005;25(19):8531-8540.

54. Aldrich MB, Chen W, Blackburn MR, Martinez-Valdez H, Datta SK, Kellems RE. Impaired germinal center maturation in adenosine deaminase deficiency. J Immunol. 2003;171(10):5562-5570

55. Jain S, Stock A, Macian F, Putterman C. A distinct T follicular helper cell subset infiltrates the brain in murine neuropsychiatric lupus. Front Immunol. 2018;9:487.

56. Ibraghimov AR, Lynch RG. T cell specialization at environmental interfaces: T cells from the lung and the female genital tract of lpr and gld mice differ from their splenic and lymph node counterparts. Eur J Immunol. 1994;24(8):1848-1852.

57. He N, Chen WL, Long KX, Zhang X, Dong GF. Association of serum CXCL13 with intrarenal ectopic lymphoid tissue formation in lupus nephritis. J Immunol Res. 2016;2016:4832543.

58. Magliozzi R, et al. Meningeal B-cell follicles in secondary progressive multiple sclerosis associate with early onset of disease and severe cortical pathology. Brain. 2007;130(pt 4):1089-1104.

59. Neyt K, Perros F, GeurtsvanKessel CH, Hammad H, Lambrecht BN. Tertiary lymphoid organs in infection and autoimmunity. Trends Immunol. 2012;33(6):297-305.

60. Hemmerich S, Butcher EC, Rosen SD. Sulfation-dependent recognition of high endothelial venules (HEV)-ligands by L-selectin and MECA 79, and adhesion-blocking monoclonal antibody. J Exp Med. 1994;180(6):2219-2226.

61. Edgar R, Domrachev M, Lash AE. Gene Expression Omnibus: NCBI gene expression and hybridization array data repository. Nucleic Acids Res. 2002;30(1):207-210.

62. Dobin A, et al. STAR: ultrafast universal RNA-seq aligner. Bioinformatics. 2013;29(1):15-21.

63. Liao Y, Smyth GK, Shi W. featureCounts: an efficient general purpose program for assigning sequence reads to genomic features. Bioinformatics. 2014;30(7):923-930.

64. Carbon S, et al. AmiGO: online access to ontology and annotation data. Bioinformatics. 2009;25(2):288-289.

65. Ben-Zvi A, et al. Mfsd2a is critical for the formation and function of the blood-brain barrier. Nature. 2014;509(7501):507-511.

66. Fischer ER, Hansen BT, Nair V, Hoyt FH, Dorward DW. Scanning electron microscopy. Curr Protoc Microbiol. 2012; 25(1):2B.2.1-2B.2.47.

67. Love MI, Huber W, Anders S. Moderated estimation of fold change and dispersion for RNA-seq data with DESeq2. Genome Biol. 2014;15(12):550.

68. Der E, et al. Single cell RNA sequencing to dissect the molecular heterogeneity in lupus nephritis. JCI Insight. 2017;2(9):93009.

69. Der E, et al. Tubular cell and keratinocyte single-cell transcriptomics applied to lupus nephritis reveal type I IFN and fibrosis relevant pathways [published online ahead of print May 20, 2019]. Nat Immunol. https://doi.org/10.1038/s41590-019-0386-1. 